Bundesgesundheitsbl - Gesundheitsforsch Gesundheitsschutz 2007 · 50:879-888 DOI 10.1007/s00103-007-0251-5

(C) Springer Medizin Verlag 2007
E. Opper · A. Worth - M. Wagner · K. Bös

Universität Karlsruhe, BRD

\section{Motorische Leistungsfähigkeit und körperlich-sportliche Aktivität von Kindern und Jugendlichen in Deutschland}


flussender Merkmale (Alter, Geschlecht, Sozialisationsmerkmale).

Bei der Interpretation der Ergebnisse verschiedener Studien ist auch $\mathrm{zu}$ berücksichtigen, dass die Kinder und Jugendlichen heute tendenziell eher größer und schwerer sind als die Altersgenossen früherer Jahre. Dies kann sich positiv auf die körperliche Leistungsfähigkeit auswirken, beispielsweise bei Testaufgaben wie Medizinballstoßen, bei denen die Körpermasse einen unmittelbaren Einfluss auf die Leistung hat, kann aber auch Nachteile haben, wenn die eigene „Körpermasse“ bewegt werden muss, z. B. beim Laufen oder Springen. Schon aus diesem Grund fällt ein Vergleich der Fitness von Kindern früher und heute nicht leicht.

Die Ergebnisse verschiedener Studien weisen dementsprechend auch unterschiedliche Tendenzen auf. Zum einen wird eine deutliche Verschlechterung der Fitness festgestellt $[6,7,8,9,10]$, zum anderen wird eine gleichbleibende motorische Leistungsfähigkeit konstatiert [11, 12]. Verbesserungen haben sich bei einigen Studien lediglich hinsichtlich ausgewählter Testübungen, nicht aber bei der Gesamtleistung der Motorik von Kindern und Jugendlichen gezeigt. Kretschmer und Giewald [11] stellen anhand eines Vergleichs der Testleistung im AST 6-11 zwischen Hamburger Grundschülern (1999) und den Referenzwerten von Bös und Wohlmann [13] lediglich in 11 von 48 Fällen eine signifikante Verschlechterung der Testleistungen fest. Diese zeigen sich in erster Linie bei der Differenzierungsfähigkeit und der Schnelligkeit. Gaschler [12] kommt nach dem Vergleich von Testdaten aus dem standardisierten und normierten Körperkoordinationstest (KTK) zu dem Schluss, dass „eine auffällige Zunahme motorisch auffälliger Kinder in den letzten 20 Jahren nicht zu beobachten" sei.

Von einer Verschlechterung der motorischen Leistungsfähigkeiten gehen Ketelhut und Bittmann [14] anhand eines quasi längsschnittlichen Vergleichs von Ergebnissen der Bundesjugendspiele aus. Auch die Ergebnisse der WIAD-AOKDSB-Studie [15] weisen auf einen Rückgang der motorischen Leistungsfähigkeit hin - hierbei wurden in Schulen in einer Querschnittsuntersuchung bundesweit
Kinder und Jugendliche zwischen 6 und 18 Jahren getestet.

Den zurzeit aktuellsten Forschungsüberblick zur motorischen Leistungsfähigkeit von Kindern und Jugendlichen hat Bös im Jahr 2003 [10] in einem Literaturreview zusammengestellt und auf dieser Grundlage auch einen Vergleich mit Daten früherer Kinder- und Jugendgenerationen hinsichtlich des Fitnesszustandes vorgenommen. In den 54 analysierten Studien von 43 Autoren aus 20 Ländern konnte er auf der Basis von 48 berechneten Vergleichen einen Rückgang der körperlichen Leistungsfähigkeit der Kinder und Jugendlichen in den untersuchten Industrieländern um 10\% im Zeitraum von 1975-200o feststellen. Dabei zeigen sich differenzielle Effekte in der Veränderung der körperlichen Leistungsfähigkeit. Bei Grundschülern sind insbesondere die Leistungen in der Gesamtkörperkoordination [7] sowie der Ausdauer [10] betroffen. Als weiteres differenzierendes Merkmal erweisen sich die Umweltbedingungen: Motorische Defizite haben besonders bei Kindern zugenommen, die in der Stadt aufwachsen. Aus verschiedenen Studien mit StadtLand-Vergleichen geht hervor, dass sich der Anteil motorisch auffälliger Kinder aus ländlichen Gebieten kaum verändert bzw. nur wenig zugenommen hat, während sich der Anteil motorisch auffälliger Kinder aus Stadtgebieten fast verdoppelt hat [7]. Einige Studien [5] weisen zudem darauf hin, dass sich eine Schere bei der körperlichen Leistungsfähigkeit erkennen lässt: Die motorisch sehr guten Kinder sind heute noch besser als die Kinder vor 30 Jahren, und die motorisch schwächeren Kinder sind noch schwächer als 1975. Gleichzeitig nimmt der Anteil der motorisch auffälligen Kinder zu. Dabei zeigt sich, dass Merkmale des Sozialstatus und des Bildungsweges nicht nur das Kognitive [16], sondern auch das Verhalten (körperlich-sportliche Aktivität) und die motorische Leistungsfähigkeit determinieren. Die Frage nach dem Zusammenhang zwischen motorischer Leistungsfähigkeit, körperlich-sportlicher Aktivität und dem sozialen Status kann mit dem Motorik-Modul (MoMo) erstmals auf repräsentativer Datenbasis beantwortet werden.

\section{Körperlich-sportliche Aktivität}

Sport in der Freizeit, sowohl in organisierter Form im Verein als auch in nicht organisierter Form, gehört nach wie vor zu den beliebtesten Freizeitbeschäftigungen von Kindern und Jugendlichen [17]. Aufgrund repräsentativer Studien aus dem angloamerikanischen Bereich, die jedoch nicht ohne weiteres auf die Situation in Deutschland zu übertragen sind, nimmt der Umfang der Aktivität bereits im Jugendalter $\mathrm{ab}[18,19,20]$. Zudem nimmt die „Indoorwelt“ zunehmend an Attraktivität zu. Hierzu gehören Computerspiele, Fernsehen sowie das Surfen im Internet [17]. Eine Studie von Bös [21] ergab, dass Kinder einen immer größeren Anteil ihrer Freizeit zu Hause vor dem Fernseher oder Computer verbringen. Ein Viertel der befragten Grundschüler im Alter von 6-10 Jahren gab sogar an, nur noch maximal einmal in der Woche im Freien zu spielen. Im Durchschnitt verbrachten die Kinder etwa 9 Stunden am Tag im Sitzen, ebenfalls 9 Stunden im Liegen, 5 Stunden stehend und lediglich eine Stunde in Bewegung [21]. Gemessen an der Bedeutung der Bewegung für die körperliche und motorische Entwicklung in den ersten Lebensabschnitten, sind die Sitz- und Stehzeiten als zu lang einzustufen $[4,10]$.

$\mathrm{Zu}$ diesem Ergebnis kommt auch die von der WHO geförderte Studie Health Behaviour in School-aged Children (HBSC): Jugendliche im Alter von 11, 13 und 15 Jahren bewegen sich zu wenig, die der HBSC-Studie zugrunde liegende Aktivitätsempfehlung zum Bewegungsverhalten von Kindern und Jugendlichen (eine Stunde pro Tag bei 5 Tagen moderater bis starker Intensität), wird nur von einem Drittel der 11- bis 15-jährigen Jungen und einem Viertel der gleichaltrigen Mädchen erfüllt. Die HBSC-Studie belegt außerdem einen signifikanten Einfluss des familiären Hintergrundes auf das Bewegungsverhalten der Jugendlichen. Jungen aus ökonomisch benachteiligten Familien bewegen sich im Vergleich zu Jungen aus der am besten gestellten Wohlstandsgruppe etwa 2-mal so häufig zu wenig. Bei Mädchen beträgt dieses Verhältnis sogar 3:1 zu Ungunsten der niedrigsten Wohlstandsgruppe [22]. 


\section{Zusammenfassung $\cdot$ Abstract}

Bundesgesundheitsbl - Gesundheitsforsch - Gesundheitsschutz 2007 · 50:879-888

DOI 10.1007/s00103-007-0251-5

(C) Springer Medizin Verlag 2007

\section{E. Opper $\cdot$ A. Worth $\cdot$ M. Wagner $\cdot$ K. Bös}

\section{Motorik-Modul (MoMo) im Rahmen des Kinder- und Jugendgesundheitssurveys (KiGGS). Motorische Leistungsfähigkeit und körperlich-sportliche Aktivität von Kindern und Jugendlichen in Deutschland}

\section{Zusammenfassung}

Motorische Leistungsfähigkeit und körperlich-sportliche Aktivität sind wesentliche Bestandteile einer gesunden Entwicklung im Kindes- und Jugendalter. Die Diagnose von motorischer Leistungsfähigkeit und körperlich-sportlicher Aktivität unterliegt jedoch bisher keinen standardisierten Kriterien, darüber hinaus liefern die untersuchten Stichproben kein repräsentatives Abbild der Gesamtbevölkerung. Die vorhandenen Daten lassen somit nur bedingt Aussagen zum Status und zur Entwicklung motorischer Merkmale und des Aktivitätsverhaltens zu. Das im Rahmen des Kinderund Jugendgesundheitssurveys (KiGGS) durchgeführte Motorik-Modul (MoMo) bietet erstmals bundesweit repräsentative Daten zur motorischen Leistungsfähigkeit und körperlich-sportlichen Aktivität von Kindern und Jugendlichen in Deutschland. Ziele des Motorik-Moduls sind die Feststellung des Ist-Zustandes von motorischer Leistungsfähigkeit und körperlich-sport- licher Aktivität sowie die Analyse der komplexen Wirkungszusammenhänge zwischen motorischer Leistungsfähigkeit, körperlich-sportlicher Aktivität und Gesundheit. Die motorische Leistungsfähigkeit wurde auf Grundlage der Systematisierung motorischer Fähigkeiten nach Bös über ein Testprofil erfasst. Hierbei wurden die Basisfähigkeiten Ausdauer, Kraft, Koordination und Beweglichkeit bzw. die ihnen untergeordneten motorischen Beschreibungskategorien über 11 (sport)motorische Tests abgebildet. Zur Erfassung der körperlichsportlichen Aktivität wurde ein 51 Items umfassender Fragebogen entwickelt. Hierbei wurden die Belastungsnormative Dauer, Intensität und Häufigkeit der Aktivität im Alltag, in der Schule, in der Freizeit und im Verein erfragt. Ergänzt wurden die genannten Teilbereiche um Fragen zur Wochenprävalenz mindestens 60-minütiger täglicher Aktivität, den materialen und örtlichen Gegebenheiten sowie zu Kogniti- on und Motivation der Aktivität. Im Rahmen des Motorik-Moduls wurden in den Jahren 2003-2006 insgesamt 4529 Kinder und Jugendliche im Alter von 4-17 Jahren an 167 Orten im gesamten Bundesgebiet hinsichtlich ihrer motorischen Leistungsfähigkeit getestet und ihrer körperlich-sportlichen Aktivität befragt. Die Hälfte aller untersuchten Kinder und Jugendlichen entstammt Familien mit mittlerem sozioökonomischem Status, ca. $15 \%$ haben einen Migrationshintergrund. Die Mehrzahl der Untersuchungsteilnehmer kommt aus einer mittelstädtischen Region, etwa ein Viertel wohnt in der Großstadt, unter 20\% sind in ländlichen Regionen angesiedelt.

\section{Schlüsselwörter}

Gesundheitssurvey · Kinder · Jugendliche · Motorische Leistungsfähigkeit · Körperlichsportliche Aktivität · Bundesweite repräsentative Studie

\section{The module „Motorik" in the German Health Interview and Examination Survey for Children and Adolescents (KiGGS). Motor Fitness and physical activity of children and young people}

\section{Abstract}

Motor fitness and physical activity are important aspects of a healthy development in childhood and adolescence. However, the assessment of motor fitness and physical activity is not subject to standardized criteria; furthermore, the samples investigated do not provide a representative image of the whole population. Therefore, the existing data only allow very limited statements on the state and development of motor fitness and physical activity. The "Motorik" module, as part of the German Health Interview and Examination Survey for Children and Adolescents (KiGGS), offers nationwide representative data on the motor fitness and physical activity of children and adolescents for the first time. Besides the baseline-analysis, another aim is to analyse the complex relationship between motor fitness, physical activity and health. Motor fitness, based on the systematisation of motor abilities, was assessed using a test profile. The test profile consists of 11 items measuring cardiorespiratory fitness, strength, coordination and mobility. Physical activity was assessed using a questionnaire containing 51 items on the duration, intensity and frequency of physical activity in everyday life, during leisure time, at school and in sports clubs. The above-mentioned questionnaire subtopics were supplemented by questions on the weekly prevalence of at least 60 minutes of daily physical activity, on material and local conditions, as well as on cognition and motivation for physical activity. In the years 2004 to 2006 , the motor fitness and physical activity of 4,529 children and young people between the ages of 4 and 17 years was investigated on 168 sample points in the context of the "Motorik" module. Half of the children and adolescents investigated belong to the middle class, approximately $15 \%$ have a background of migration. The majority of the subjects come from small towns, about a quarter live in the city, less than $20 \%$ are settled in rural areas.

\section{Keywords}

Health survey · Children · Adolescents .

Motor fitness · Physical activity .

Representative data 
Bei einer repräsentativen Studie mit luxemburgischen Kindern und Jugendlichen im Alter von 9, 14 und 18 Jahren hat sich gezeigt, das nur knapp ein Fünftel (22\%) der Schüler und Schülerinnen die Mindestanforderung im Hinblick auf körperlich-sportliche Aktivität erfüllt. Hier wurde allerdings schon die Richtlinie von 6o Minuten pro Tag und nicht mehr nur eine Aktivität an 5 Tagen zugrunde gelegt [23]. Die NASPE [24], das President's Council on Physical Fitness \& Sports [25], das Australian Government Department of Health and Ageing [26] und die WHO [27] fordern inzwischen, dass Kinder täglich 6o Minuten bei moderater bis starker Intensität aktiv sein sollen.

Ergebnisse der European Youth Heart Study der skandinavischen Forscher um L.B. Andersen und K. Froberg [28] zeigen, dass sogar die bislang gültige 60Minuten-Empfehlung zu gering ist, um den besten Schutz vor Herz-KreislaufErkrankungen zu erzielen. Die Forscher haben festgestellt, dass etwa 90 Minuten körperlich-sportliche Aktivität am Tag den besten Schutz bieten. Bei 9-jährigen Kindern zeigen sich die besten Werte bei einer täglichen mäßigen bis starken Aktivität. Bei Jugendlichen genügen $88 \mathrm{Mi}$ nuten tägliche Aktivität, um den besten Schutz vor Herz-Kreislauf-Erkrankungen zu erzielen.

Mit Blick auf die Möglichkeiten gesundheitlicher Prävention durch körperlich-sportliche Aktivität wird es ein wichtiges Ziel sein, die Activity-Guideline neu zu definieren. Fest steht, dass sich damit die Zahl der Jungen und Mädchen und damit auch die der Kinder und Jugendlichen des MoMo, die diese Richtlinie nicht erfüllen, weiter erhöhen wird.

Die epidemiologischen Informationen über das Ausmaß und den Entwicklungsverlauf von körperlich-sportlicher Aktivität in den verschiedenen Subpopulationen sind auch aus einer interventionsorientierten Sichtweise wichtig, um „inaktive“ Zielgruppen zu identifizieren. Notwendig ist darüber hinaus die möglichst genaue Kenntnis der Determinanten, die für die Herausbildung und Aufrechterhaltung des Sportverhaltens relevant sind. Dabei sind sowohl die internen (körperliche, kognitive oder emotionale) Determinanten [29] als auch die externen (soziale oder struk- turelle) Faktoren [30] zu berücksichtigen. Ein Überblick über die potenziellen Determinanten des Sport- und Bewegungsverhaltens bei Kindern und Jugendlichen findet sich bei Sallis und Owen [31] und auch bei Dishman et al. [32].

Ein aktueller Überblick über die vorhandenen nationalen Daten kommt zu dem Schluss, dass sich die körperlichsportliche Aktivität von Kindern und Jugendlichen in den vergangenen 25 Jahren kaum verändert hat [33]. Insgesamt zeigt die Forschung in dieser Frage jedoch ein uneinheitliches Bild - die Kennzeichnung der Situation reicht vom „Aktivitätsboom“ bis hin zur „Aktivitätskrise“. Beide Aussagen sind jeweils mit methodologischen Problemen behaftet, die sich vor allem auf die Frage des Erhebungsinstrumentes und die zugrunde gelegten Stichproben beziehen. So liegen bislang keine bundesweit repräsentativen Daten zur körperlich-sportlichen Aktivität von Kindern und Jugendlichen vor. Die vorhandenen Daten basieren auf anfallenden Stichproben und erheben allenfalls den Anspruch, auf kommunaler oder Länderebene repräsentativ zu sein [34, 35], oder sie sind in einem anderen Kontext geplant und erfassen nur einen geringen Ausschnitt des körperlichen Aktivitätsverhaltens - z. B. nur die sportliche und nicht die körperliche Alltagsaktivität wie in der Shell-Studie [17].

Das MoMo und der Kinder- und Jugendgesundheitssurvey (KiGGS) stellen erstmals umfassend belastbare Daten zur körperlich-sportlichen Aktivität der heranwachsenden Generation in Deutschland bereit.

\section{MoMo-Untersuchungskonzept}

Das MoMo ist ein Teilmodul des KiGGS (Kern-Survey) des Robert Koch-Institutes (RKI). In den Jahren 2003-2006 haben Forscher des MoMo, das vom Bundesministerium für Familie, Senioren, Frauen und Jugend gefördert wird, bundesweit 4529 Kinder und Jugendliche zwischen 4 und 17 Jahren hinsichtlich ihrer motorischen Leistungsfähigkeit getestet und zu ihrer körperlich-sportlichen Aktivität befragt. Damit liegt erstmals eine bundesweit repräsentative Stichprobe zur motorischen Leistungsfähigkeit und der körperlich-sportlichen Aktivität von Kindern und Jugendlichen vor.

Die Kooperation von MoMo und KiGGS ermöglicht eine Forschungsperspektive, die in der interdisziplinären Verknüpfung motorischer, medizinischer und subjektiver Gesundheitsparameter über den bisherigen gesundheits- und sportwissenschaftlichen Forschungsstand hinaus reicht.

\section{Untersuchungsziele}

Das erste Ziel des MoMo ist es, die Ist-Situation bezüglich motorischer Leistungsfähigkeit und körperlich-sportlicher Aktivität von Kindern und Jugendlichen zu analysieren. Auf Grundlage der repräsentativen Datenbasis wird damit eine Baseline geschaffen bzw. Normwerte für die Einordnung der motorischen Leistungsfähigkeit und des Sportverhaltens von Heranwachsenden festgelegt. Mit diesen Ausgangsdaten kann die motorische Entwicklung und das Sportverhalten zukünftig eingeordnet und in Beziehung gesetzt werden. Damit ist die Basis für eine langfristige Aktivitäts- und Fitnessberichterstattung geschaffen. Ein weiteres wichtiges Ziel des MoMo ist die Analyse der Zusammenhänge zwischen körperlicher - und im Besonderen - sportlicher Aktivität, motorischer Leistungsfähigkeit und Gesundheit bzw. Gesundheitsverhalten. Diese Zusammenhänge können durch eine Verknüpfung mit den KiGGS-Daten erstmals belastbar analysiert werden. Weiterhin geht es auch darum, differenzierende Merkmale, wie z. B. den sozialen Status der Familie, den Migrationshintergrund und Unterschiede entlang der Wohnortgröße herauszuarbeiten.

Die Ergebnisse der oben genannten Analyse liefern zudem eine wichtige empirische Grundlage, um Förderprogramme zur Bewegung und Aktivität in unterschiedlichen Settings, z. B. in Kindergärten, Schulen und Vereinen, entwickeln zu können. Dazu gehört auch die Entwicklung von Informationsmaterial für Eltern, Lehrer und Erzieher sowie das Angebot von Informationsveranstaltungen. 
Tabelle 1

Erfassung der Motorik-Dimensionen im Kinder- und Jugendgesundheitssurvey (KiGGS) und Motorik-Modul (MoMo)

\begin{tabular}{|c|c|c|c|c|c|}
\hline \multirow[b]{2}{*}{ Testbereiche } & \multirow[b]{2}{*}{ Testinhalt } & \multirow[b]{2}{*}{ Testaufgaben } & \multicolumn{3}{|c|}{ Altersgruppen } \\
\hline & & & 4-5 Jahre & 6-10 Jahre & 11-17 Jahre \\
\hline \multirow[t]{5}{*}{ Koordination } & KP & Linie nachfahren (MLS) & $x$ & $x$ & $\mathbf{x}$ \\
\hline & KP & Einbeinstand & $x$ & $x$ & $\mathbf{x}$ \\
\hline & KP & Balancieren rückwärts & $\mathbf{x}$ & $\mathbf{x}$ & $\mathbf{x}$ \\
\hline & $\mathrm{KZ}$ & Stifte einstecken (MLS) & $x$ & $\mathrm{x}$ & $\mathrm{x}$ \\
\hline & RS & Reaktionstest & $\mathrm{x}$ & $\mathrm{x}$ & $\mathbf{x}$ \\
\hline \multirow[t]{4}{*}{ Kraft } & KA & Seitliches Hin- und Herspringen & $\mathrm{x}$ & $x$ & $x$ \\
\hline & KA & Liegestütz & - & $\mathbf{x}$ & $\mathbf{x}$ \\
\hline & SK & Standweitsprung & $\mathbf{x}$ & $\mathbf{x}$ & $\mathbf{x}$ \\
\hline & SK & Kraftmessplatte & $\mathbf{x}$ & $\mathbf{x}$ & $\mathbf{x}$ \\
\hline Ausdauer & AA & Fahrradausdauertest & - & $x$ & $\mathrm{x}$ \\
\hline Beweglichkeit & B & Rumpfbeugen & $\mathrm{X}$ & $x$ & $\mathbf{x}$ \\
\hline
\end{tabular}

Nicht hervorgehoben: KiGGS-Kern-Survey (Robert Koch-Institut), hervorgehoben bzw. fett: Zusatzmodul Motorik (MoMo); KP Koordination bei Präzisionsaufgaben, KZ Koordination unter Zeitdruck, RS Reaktionsschnelligkeit, KA Kraft-Ausdauer, SK Schnellkraft, AA Aerobe Ausdauer, B Beweglichkeit, MLS Motorische Leistungsserie (Kurzform nach Sturm \& Büssing)

\section{Untersuchungsmethoden}

Die motorische Leistungsfähigkeit wurde auf Grundlage der Systematisierung motorischer Fähigkeiten nach Bös [36] über ein Testprofil mit 11 Testitems zur Ausdauer, Kraft, Koordination und Beweglichkeit gemessen [37]. Die körperlich-sportliche Aktivität wurde mit einem selbst entwickelten Fragebogen erfasst.

\section{Motorische Leistungsfähigkeit}

Im KiGGS wurden bei den 4- bis 10-Jährigen die Dimensionen Koordination, Kraft und Beweglichkeit und bei den 11bis 17-Jährigen die Ausdauerleistungsfähigkeit berücksichtigt. Erste ausgewählte Ergebnisse dazu finden sich in dieser $\mathrm{Pu}$ blikation [37]. In der Teilstichprobe des MoMo werden diese Dimensionen für die jeweiligen Altersgruppen komplementär ergänzt (• Tabelle 1), sodass bei der Stichprobe des MoMo für die getesteten Kinder und Jugendlichen Daten zu allen 11 Testaufgaben vorliegen.

Die Auswahl der Testübungen erfolgt unter anderem vor dem Hintergrund, dass die Tests von den Kindern zwischen 4 und 17 Jahren durchführbar sind, um mögliche Alterseffekte feststellen zu können. Dies ist bei 9 Testaufgaben möglich, lediglich die Liegestütze und den Fahrradausdauertest haben die 4- bis 5-Jährigen nicht absolviert.
Die MoMo-Ergebnisse zur motorischen Leistungsfähigkeit werden in der Basispublikation des Moduls [38] publiziert.

\section{Körperlich-sportliche Aktivität}

Die körperlich-sportliche Aktivität wurde im MoMo mit selbst entwickelten Fragebögen erfasst. Die 4- bis 10-jährigen Kinder wurden bezüglich der körperlich-sportlichen Aktivität interviewt. Sie beantworteten die Fragen mit Unterstützung ihrer Eltern. Die 11- bis 17Jährigen haben den Fragebogen selbst ausgefüllt. Wie auch bei der motorischen Leistungsfähigkeit, sind die Fragen für alle Altersgruppen deckungsgleich. Der Unterschied besteht lediglich im Bezug zum Setting. Bei den 4- bis 5-Jährigen wurde nach der sportlichen Aktivität im Kindergarten und bei den 6- bis 17Jährigen nach der Sportaktivität in der Schule gefragt. Ein weiterer Unterschied besteht darin, dass die 6- bis 17-Jährigen Fragen zum angenommenen Nutzen durch körperlich-sportliche Aktivität sowie zu den Motiven des Sporttreibens beantworteten. In $\bullet$ Tabelle 2 sind die verschiedenen Inhaltsbereiche des MoMo-Fragebogens abgebildet.

Im KiGGS wurden Eckdaten zur körperlich-sportlichen Aktivität erhoben (- Tabelle 3). Die diesbezüglichen Ergebnisse finden sich im Beitrag von T. Lam- pert et al. in diesem Heft [39]. Die MoMo-Ergebnisse zu körperlich-sportlicher Aktivität werden in der Basispublikation des Moduls [38] publiziert.

\section{Testgüte der Testinstrumente}

Die eingesetzten Motoriktests und der Fragebogen zur Erfassung der körperlich-sportlichen Aktivität wurden im Rahmen von Pretests und Zusatzstudien mittels verschiedener Instrumente überprüft [40].

\section{Motorische Tests}

Im Rahmen der Pretests wurden die für die 3 Altersklassen ausgewählten Tests mittels der Test-Retest-Methode überprüft. Die Tests wurden nach 4 Tagen mit den gleichen Kindern, dem gleichen Testleiter und der gleichen Testsituation ein zweites Mal durchgeführt. Zur Überprüfung der Objektivität wurden die Kinder zum ersten Messzeitpunkt gleichzeitig von 2 Testleitern untersucht. Die Objektivität ist mit Korrelationen von 0,98-0,99 sehr gut. Es zeigen sich in keinem Fall signifikante Mittelwertsunterschiede. Die prozentuale Differenz liegt bei allen Tests unter einem Prozentpunkt. Die Messwertaufnahme ist demnach unabhängig vom Versuchsleiter, und für die ausgewählten Testaufgaben ist eine hohe Objektivität gegeben. 
Tabelle 2

Erfassung der körperlich-sportlichen Aktivität im Motorik-Modul (MoMo). Altersklasse der 4- bis 6-Jährigen (Kindergartenkinder, insgesamt 29 Fragen), Altersklasse der 6- bis 17-Jährigen (insgesamt 51 Fragen)

\section{Inhaltlicher Bereich Fragen}

Körperlich sportliche Aktivität allgemein An wie vielen der letzten 7 Tage warst du für mindestens 60 min am Tag körperlich aktiv? (2 Fragen)

\begin{tabular}{ll}
$\begin{array}{l}\text { 4-6 Jahre Sportliche Aktivität im } \\
\text { Kindergarten (4 Fragen), } \\
\text { 6-17 Jahre Sportliche Aktivität } \\
\text { in der Schule (7 Fragen) }\end{array}$ & 4-6 Jahre Differenzierung nach Häufigkeit, Dauer, Intensität \\
\hline $\begin{array}{l}\text { Körperliche Aktivität im Alltag (4 Fragen) } \\
\text { 6-17 Jahre Differenzierung nach Häufigkeit, Dauer, Intensität, Art der Bewegung/Sportarten }\end{array}$ & Schulweg, Spielen im Freien, Gartenarbeit, Fußwege \\
$\begin{array}{l}\text { Verfügbarkeit von Sportstätten und } \\
\text { Geräten (2 Fragen) }\end{array}$ & Sportstätten, Geräte \\
\hline $\begin{array}{l}\text { Sportliche Aktivität in der Freizeit } \\
\text { organisiert im Verein (7 Fragen) }\end{array}$ & $\begin{array}{l}\text { Differenzierung nach Häufigkeit, Dauer, Intensität, Art der Bewegung/Sportarten, } \\
\text { Wettkampfteilnahme }\end{array}$ \\
$\begin{array}{l}\text { Sportliche Aktivität in der Freizeit } \\
\text { (außerhalb des Vereins) (6 Fragen) }\end{array}$ & Differenzierung nach Häufigkeit, Dauer, Intensität, Art der Bewegung/Sportarten \\
$\begin{array}{l}\text { Sportverhalten der Bezugspersonen/ } \\
\begin{array}{l}\text { Peergroup (4 Fragen) } \\
\text { 6-17 Jahre Angenommener Nutzen/ }\end{array}\end{array}$ & Eltern, Geschwister, Freunde \\
$\begin{array}{l}\text { Effekte körperlich-sportlicher Aktivität } \\
\text { (9 Fragen) }\end{array}$ & \\
$\begin{array}{l}\text { 6-17 Jahre Motive zum Sporttreiben } \\
\text { (10 Fragen) }\end{array}$ & Ich treibe Sport um ... (z. B.... um etw. für meine Figur zu tun) \\
\hline
\end{tabular}

Tabelle 3

Erfassung der körperlich-sportlichen Aktivität im Kinder- und Jugendgesundheitssurvey (KiGGS)
Altersklasse
3-6 Jahre (Frage 62, wird durch Befragung der Eltern erfasst) (3 Fragen) Wie häufig...
- spielt Ihr Kind im Freien?
- treibt Ihr Kind Sport im Verein?
- treibt Ihr Kind Sport außerhalb des Vereins?

7-10 Jahre (Frage 64, wird durch Befragung der Eltern erfasst) (3 Fragen) Wie häufig...

- spielt Ihr Kind im Freien?

- treibt Ihr Kind Sport im Verein?

- treibt Ihr Kind Sport außerhalb des Vereins?

11-13 Jahre (Frage 32, wird vom Jugendlichen direkt beantwortet). Wie oft bist du in deiner Freizeit körperlich aktiv? So, dass du richtig ins Schwitzen kommst oder außer Atem bist

14-17 Jahre (Frage 43, wird vom Jugendlichen direkt beantwortet) Wie oft bist du in deiner Freizeit körperlich aktiv? So, dass du richtig ins Schwitzen kommst oder außer Atem bist

Für die Reliabilität der motorischen Tests zeigen sich durchweg gute $(r>0,7$, Mittelwertsunterschied signifikant) bis sehr gute $(r>0,7$, kein signifikanter Mittelwertsunterschied, $\mathrm{p}>0,05)$ TestRetest-Reliabilitätskoeffizienten in der Gesamtstichprobe. Die Gesamtreliabilität wurde über den standardisierten Gesamtwert berechnet. Hier ergibt sich eine Korrelation von $\mathrm{r}=0,97(\mathrm{p}=0,00)$ und kein signifikanter Mittelwertsunter- schied $(p=0,28)$. Das Testinstrumentarium erweist sich als überaus zuverlässig und präzise.

Die inhaltliche Validität der motorischen Tests wurde durch ein Expertenrating abgesichert. Insgesamt wurden gute Noten hinsichtlich Aussagekraft und Durchführbarkeit vergeben.

\section{Körperlich-sportliche Aktivität}

Im Dezember 2003 wurde der Fragebogen hinsichtlich seiner Reliabilität (TestRetest) und seiner Validität überprüft. Die Validierung erfolgte mittels eines Schrittzählers sowie mittels Herzfrequenzmessungen [40].

Der Test-Retest wurde im Abstand von 7 Tagen durchgeführt. Für die Reliabilität des gesamten Fragebogens wurde ein Korrelationskoeffizient von $r=0,97$ 
berechnet. Auch hier wurde kein signifikanter Mittelwertsunterschied festgestellt. Die Reliabilität verbessert sich somit mit zunehmendem Alter.

Für die Überprüfung mittels Schrittzähler ergab die Untersuchung einen Korrelationskoeffizienten von $r=0,26$. Bei der Überprüfung mittels Pulsuhr wurde $r=0,28$ für intensive Aktivität und $r=0,51$ für moderate Aktivität festgestellt. Das Ergebnis bestätigt die Erwartung, dass „intensive Aktivitäten“ aussagekräftiger sind als „wenig intensive Aktivitäten“.

Im Februar 2006 erfolgte eine weitere Validierungsstudie mittels SenseWear-Armband [41]. Für den Bereich des Vereinssports ergab die Studie einen Zusammenhang von $\mathrm{r}=0,66$ und für den nicht organisierten Freizeitsport einen Zusammenhang von $\mathrm{r}=0,56$. Der gemessene Zusammenhang zwischen Aktivitätsfragebögen und Schrittzähler, Pulsmessungen bzw. SenseWear-Armband bestätigt die Aussagekraft der gewählten Befragungsmethode.

\section{Untersuchungsstichprobe}

Die Stichprobe des MoMo umfasst 4529 Mädchen und Jungen von 4-17 Jahren. Die Kinder und Jugendlichen wurden in der Zeit von Juni 2003 bis Juni 2006 in insgesamt 167 Orten in ganz Deutschland getestet (• Abb. 1).

Die Stichprobenziehung erfolgte durch das RKI nach Vorgaben des Zentrums für Umfragen, Methoden und Analysen (ZUMA) in Mannheim.

Um repräsentative Aussagen treffen zu können, wurde für die Analyse ein Gewichtungsfaktor berechnet, der Abweichungen der Netto-Stichprobe von der Bevölkerungsstruktur (Stand 31.12.2004) hinsichtlich Alter (in Jahren), Geschlecht, Region (Ost/West/Berlin) und Staatsangehörigkeit korrigiert.

Für das Motorik-Modul zeigt sich in allen Altersgruppen eine gleichmäßige Geschlechterverteilung (• Tabelle 4, 5). Insgesamt haben $51,1 \%$ Jungen $(\mathrm{N}=2314)$ und 48,9\% Mädchen $(\mathrm{N}=2215)$ teilgenommen, was einer Spiegelung der Gesamtpopulation entspricht.

Zur weiteren Auswertung werden die Kinder und Jugendlichen in 3 Altersgruppen zusammengefasst: die Kindergarten-

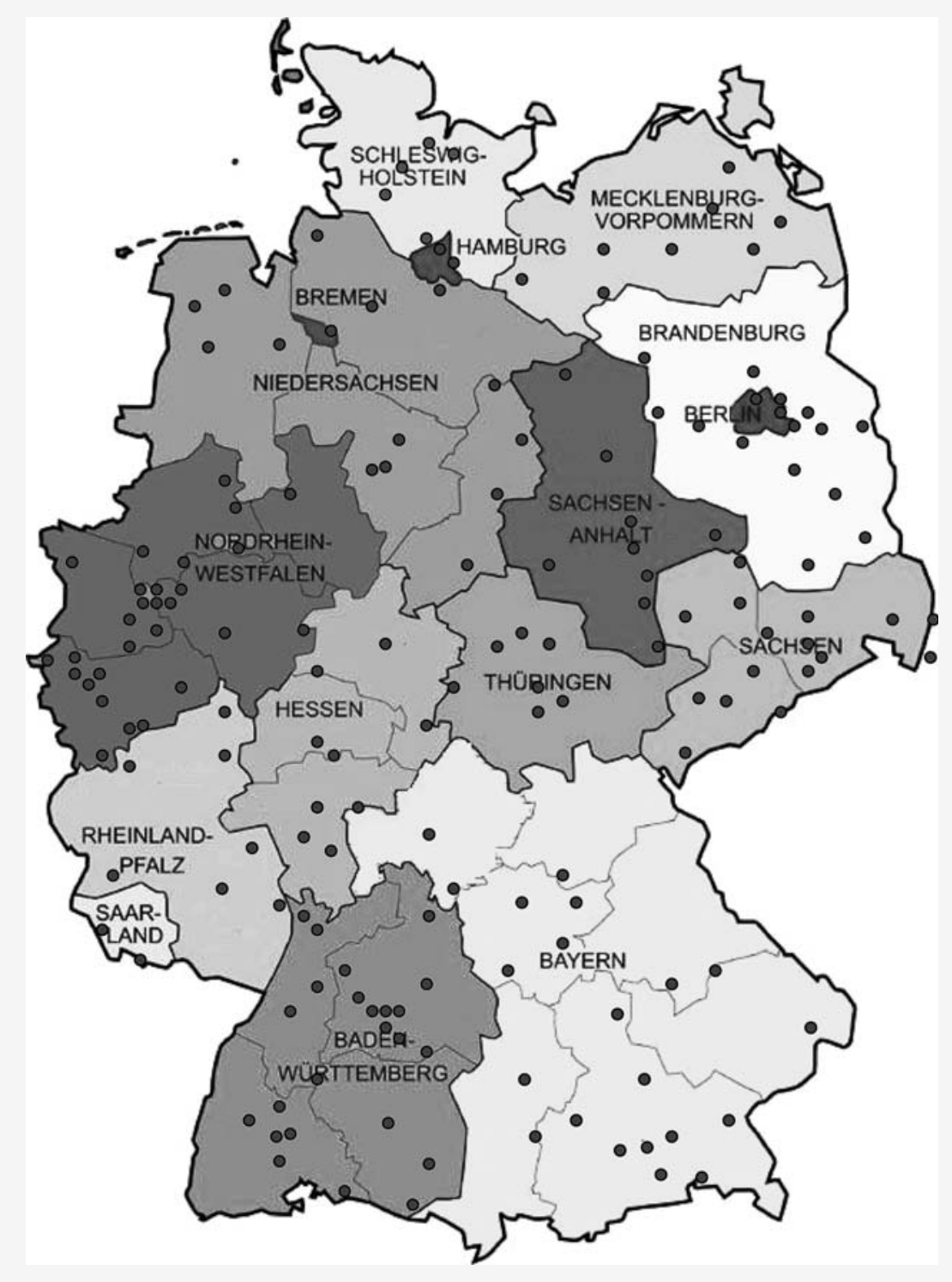

Abb. $1 \Delta$ Deutschlandkarte mit den Untersuchungsorten

kinder (4-5 Jahre, 13,3\%), die Grundschüler (6-10 Jahre, 33,7\%) und die Schüler der Sekundarstufe (11-17 Jahre, $53 \%$ ).

\section{Sozialstatus}

„In der KiGGS-Studie wird mit dem sozialen Status ein Konzept sozialer Ungleichheit herangezogen, das erlaubt die relative Position der Kinder und Jugendlichen sowie deren Familien im gesellschaftlichen Ungleichheitsgefüge $\mathrm{zu}$ bestimmen. Gemessen wird der soziale Status anhand von Angaben der Eltern zu ihrer Schulbildung und beruflichen Qualifikation, ihrer beruflichen Stellung und zum Haushaltsnettoeinkommen. [...]
$\mathrm{Zu}$ berücksichtigen ist dabei, dass es sich bei den so ermittelten Statusgruppen um statistische Konstrukte handelt. Die untere Statusgruppe sollte deshalb nicht mit Unterschicht oder Armut gleichgesetzt werden. Ebenso wenig repräsentiert die obere Statusgruppe die gesellschaftliche Oberschicht." [42]

Bei einer Betrachtung der MoMoStichprobe nach dem Sozialstatus zeigt sich, dass mit 47,4\% (N=2119) der Großteil der Kinder aus Familien mit einem mittleren Sozialstatus kommt. 25\% ( $\mathrm{N}=1115)$ der Heranwachsenden stammen aus $\mathrm{Fa}$ milien mit einem niedrigen und 27,6\% $(\mathrm{N}=1232)$ aus Familien mit einem hohen Sozialstatus. 
Tabelle 4

Untersuchungsstichprobe des Motorik-Moduls (MoMo) nach Alter und Geschlecht ( 4 bis 10 Jahre) und gesamt

\begin{tabular}{|c|c|c|c|c|c|c|c|}
\hline \multirow[t]{2}{*}{ Geschlecht } & \multicolumn{7}{|c|}{ Altersklassen } \\
\hline & 4 & 5 & 6 & 7 & 8 & 9 & 10 \\
\hline $\mathrm{m}$ & 153 & 155 & 158 & 161 & 158 & 152 & 156 \\
\hline w & 146 & 146 & 149 & 153 & 151 & 147 & 142 \\
\hline Gesamt & 299 & 301 & 307 & 314 & 309 & 299 & 298 \\
\hline
\end{tabular}

Tabelle 5

Untersuchungsstichprobe des Motorik-Moduls (MoMo) nach Alter und Geschlecht (11 bis 17 Jahre) und gesamt

\begin{tabular}{|c|c|c|c|c|c|c|c|c|c|}
\hline \multirow[t]{2}{*}{ Geschlecht } & \multicolumn{9}{|c|}{ Altersklassen } \\
\hline & 11 & 12 & 13 & 14 & 15 & 16 & 17 & $\begin{array}{l}\text { Gesamt } \\
\text { (4-17 J.) }\end{array}$ & $\%$ (gesamt) \\
\hline $\mathrm{m}$ & 157 & 158 & 174 & 180 & 186 & 194 & 172 & 2.314 & $51,1 \%$ \\
\hline w & 154 & 154 & 160 & 179 & 176 & 178 & 178 & 2.215 & $48,9 \%$ \\
\hline Gesamt & 311 & 312 & 334 & 359 & 362 & 372 & 350 & 4.529 & $100 \%$ \\
\hline
\end{tabular}

Zwischen Mädchen und Jungen zeigen sich hinsichtlich des Sozialstatus kaum Unterschiede: 25,8 \% der Mädchen und $24,3 \%$ der Jungen leben in Familien mit einem niedrigen und $26,9 \%$ der befragten Mädchen und 28,3\% der Jungen in Familien mit einem hohen Sozialstatus. Mit 47,7\% sind die meisten Mädchen und Jungen ( $47,5 \%)$ einem mittleren sozialen Status zugehörig.

Bei der Betrachtung der 3 Altersgruppen zeigen sich ebenfalls keine Unterschiede. Auch hier kommen die meisten Kinder in allen Altersgruppen aus Familien mit einem mittleren Sozialstatus (45 Jahre: $45 \%$, 6-10 Jahre: $46,8 \%, 11-17$ Jahre: $48,5 \%)$.

\section{Migrationshintergrund}

„Als Migranten werden Kinder und Jugendliche bezeichnet, die selbst aus einem anderen Land zugewandert sind und von denen mindestens ein Elternteil nicht in Deutschland geboren ist oder von denen beide Eltern zugewandert bzw. nicht deutscher Staatsangehörigkeit sind." [43]

$14,4 \%(N=648)$ der Kinder und Jugendlichen haben einen Migrationshintergrund. Auch hier ist die Geschlechterverteilung mit $14,9 \%$ der untersuchten Mädchen und 13,9\% der Jungen annähernd ausgeglichen.
Bei der Betrachtung der Altersgruppen zeigt sich, dass die 4- bis 5 -Jährigen mit 15,9\% einen etwas größeren Migrationsanteil aufweisen. Bei den 6- bis 10Jährigen haben $14,0 \%$ und bei den 11- bis 17-Jährigen 14,4 \% einen Migrationshintergrund.

Bei der Betrachtung des Migrationshintergrundes nach Alter und Geschlecht zeigt sich, dass bei den Jungen der Anteil in der jüngsten Altersgruppe mit 19,5\% am höchsten ist und bis auf $12,4 \%$ bei den 11- bis 17-Jährigen sinkt. Bei den Mädchen verhält es sich genau umgekehrt: In der jüngsten Altersgruppe sind 12,1\% Migrantinnen, und dieser Anteil steigt bis auf

\section{Wohnortgröße}

Hinsichtlich der Region zeigt sich, dass mit 29,4\% die meisten Kinder und Jugendlichen einer mittelstädtischen Region zuzuordnen sind (20.000 bis unter 100.00o Einwohner). 27,9\% kommen aus einer kleinstädtischen Region (5000 bis unter 20.000 Einwohner), 24,2 \% aus einer großstädtischen Region (100.000 und mehr Einwohner) und 18,5\% leben in einer ländlichen Region (weniger als 5000 Einwohner).

Bei einer geschlechtsdifferenzierenden Betrachtung zeigt sich ebenfalls, dass die 16,1\% bei den 11-17 Jahre alten Mädchen. meisten Mädchen (28,7\%) und Jungen (30,1\%) in einer mittelstädtischen Region leben.

Dies trifft auch bei einer Betrachtung der 3 Altersgruppen zu, denn sowohl von den 4- bis 5-Jährigen (30,1\%), den 6- bis 10-Jährigen $(29,1 \%)$ und auch den 11- bis 17-Jährigen $(29,3 \%)$ leben die meisten Kinder in einer mittelstädtischen Region.

Die Deskription der Ergebnisse des MoMo ist derzeit in Vorbereitung [37]. Dabei wird die Frage nach dem Ist-Zustand der motorischen Leistungsfähigkeit von Kindern und Jugendlichen in Deutschland beantwortet und differenziert nach unterschiedlichen Fähigkeiten sowie nach den oben dargestellten Moderatorvariablen Alter, Geschlecht, Migrationshintergrund und Stadt/Land Sozialstatus dargestellt. Weiterhin wird die Prävalenz der körperlich-sportlichen Aktivität von Kindern und Jugendlichen beschrieben und ebenfalls differenziert nach Alter, Geschlecht, Sozialstatus und nach unterschiedlichen Bereichen (z. B. Verein, Schule, unorganisierter Sport in der Freizeit) analysiert.

\section{MoMo - Ausblick}

Zusammenfassend lässt sich festhalten, dass die bisher vorhandene Datenlage der verschiedenen Einzelstudien für einen Rückgang der körperlichen Leistungsfähigkeit von Kindern und Jugendlichen spricht. Eine endgültige, exakte Beurteilung von Kohorteneffekten bzw. eines zeitlichen Wandels in der Veränderung der motorischen Leistungsfähigkeit von Kindern und Jugendlichen war im strengen methodologischen Sinne bisher jedoch nicht möglich, da der Vergleich mit einer bundesweiten, repräsentativen Eichstichprobe aussteht. Diese Eichstichprobe ist nun mit den Daten des MoMo gewonnen. Auf der Grundlage dieser Datenbasis wird es möglich, Normwerte für die motorische Leistungsfähigkeit von Kindern und Jugendlichen zwischen 4 und 17 Jahren zu erstellen.

Die MoMo-Daten sind demnach die Grundlage für eine langfristige Beobachtung und Analyse der Veränderung der motorischen Leistungsfähigkeit, da diese Daten bei zukünftigen Untersuchungen als Referenz herangezogen werden kön- 
nen. Mit dem MoMo-Testsystem liegt zudem ein Testinstrumentarium vor, auf dessen Basis sich künftig Teststandards definieren und Empfehlungen für die Entwicklung von Testaufgaben und Testbatterien formulieren lassen. Auch bezüglich der körperlich-sportlichen Aktivität können nun bundesweit repräsentative Aussagen für Kinder und Jugendliche getroffen werden. Die Verknüpfung mit dem KiGGS-Datensatz ermöglicht es außerdem, erstmals Zusammenhänge zwischen der motorischen Leistungsfähigkeit, der körperlich-sportlichen Aktivität und Gesundheit zu analysieren.

Die zu erwartenden Befunde sind neben ihrer gesundheits- und sportwissenschaftlichen Bedeutung auch von hoher praktischer und gesundheitspolitischer Relevanz. Praxisbedeutsam - z. B. im Hinblick auf die Entwicklung von Bewegungsförderprogrammen und einer gesundheitsrelevanten Gestaltung von Sport im Kindergarten, in der Schule und im Verein - ist die Klärung, welche bedeutsamen Zusammenhänge zwischen körperlich-sportlicher Aktivität und der motorischen Leistungsfähigkeit, Gesundheit bzw. Gesundheitsverhalten bestehen, welcher Sport unter welchen Bedingungen wirksam sein kann und welche Zusammenhänge mit dem Schulsport vorliegen. Antworten darauf sind darüber hinaus eine Grundlage für die politische Diskussion zur Rolle körperlich-sportlicher Aktivität im Rahmen öffentlicher Gesundheitsförderung, z. B. in Bezug auf bevölkerungsbezogene Maßnahmen zur Förderung körperlich-sportlicher Aktivitäten.

\section{Korrespondierende Autorin}

\section{Dr. Elke Opper}

Institut für Sport und Sportwissenschaft der Universität Karlsruhe

Kaiserstraße 12

76131 Karlsruhe, BRD

E-Mail: opper@sport.uka.de

\section{Literatur}

1. Zimmer R (1993) Handbuch Bewegungserziehung. Didaktisch-methodische Grundlagen und Ideen für die Praxis. Herder, Freiburg
2. Zimmer R (1999) Handbuch der Psychomotorik. Theorie und Praxis der psychomotorischen Förderung von Kindern. Herder, Freiburg

3. Fuchs R (1997) Psychologie und körperliche Aktivität. Hogrefe, Göttingen

4. Opper E, Worth A, Bös K (2005) Kinderfitness Kindergesundheit. Bundesgesundheitsbl Gesundheitsforsch Gesundheitsschutz 48:854-862

5. Woll A, Bös K (2004) Körperlich-sportliche Aktivität und Gesundheit von Kindern. In: Woll A, Bös K (Hrsg) Kinder Bewegen. Hörner, Forst, S 8-21

6. Dordel S (1998) Ätiologie und Symptomatik motorischer Defizite und Auffälligkeiten. In: Bundeszentrale für gesundheitliche Aufklärung BzGA (Hrsg) Gesundheit von Kindern - Epidemiologische Grundlagen. Band 3: Forschung und Praxis in der Gesundheitsförderung, 2. Aufl. BZgA, Köln, S 49-59

7. Dordel S (2000) Kindheit heute:Veränderte Lebensbedingungen=reduzierte motorische Leistungsfähigkeit? Motorische Entwicklung und Leistungsfähigkeit im Wandel. Sportunterricht 49:341-349

8. Klaes L, Rommel A, Cosler D, Zens YCK (2000) Bewegungsstatus von Kindern und Jugendlichen in Deutschland. Kurzfassung einer Untersuchung im Auftrag des Deutschen Sportbundes und der AOKDie Gesundheitskasse. WIAD, Bonn

9. Bös K, Opper E, Woll A (2002) Fitness in der Grundschule - ausgewählte Ergebnisse. Haltung und Bewegung 22:5-20

10. Bös K (2003) Motorische Leistungsfähigkeit von Kindern und Jugendlichen. In: Schmidt W, Hartmann-Tews I, Brettschneider W-D (Hrsg) Erster Deutscher Kinder- und Jugendsportbericht. Hofmann, Schorndorf, S 85-109

11. Kretschmer J, Giewald C (2001) Veränderte Kindheit - veränderter Schulsport? Sportunterricht 50:36-42

12. Gaschler P $(1999,2000,2001)$ Motorik von Kindern und Jugendlichen heute - eine Generation von „Weicheiern, Schlaffis und Desinteresse"? (Teil 1, Teil2, Teil 3). Haltung und Bewegung 19(3):5-16; 20(1):5-16; 21(1):5-17

13. Bös K, Wohlmann R (1987) Allgemeiner Sportmotorischer Test (AST 6-11) zur Diagnose der konditionellen und koordinativen Leistungsfähigkeit. Lehrhilfen Sportunterricht 36:145-160

14. Ketelhut K, Bittmann F (1999). Bewegungsmangel im Kindesalter. Sind Gesundheit und Fitness heutiger Kinder besorgniserregend? Sportunterricht 50:342-344

15. Klaes L, Rommel A, Cosler D, Zens YCK (2003) Kurzfassung der WIAD-Studie II: Bewegungsstatus von Kindern und Jugendlichen in Deutschland. Deutscher Sportbund, Frankfurt/Main

16. Prenzel M, Baumert J, Blum W et al. (2003) Pisa 2003 - Ergebnisse des zweiten internationalen Vergleichs. Zusammenfassung. PISA-Konsortium Deutschland

17. Deutsche Shell Holding GmBH (2002) Jugend 2002. 14. Shell Jugendstudie. Fischer, Frankfurt am Main

18. Rowland TW (1990) Exercise and children's health. Human Kinetics Books, Champaign, IL

19. Torun, B, Davies PSW, Livingstone MBE et al. (1996) Energy requirements and dietary energy recommendations for children and adolescents 1 to 18 years old. Eur J Clin Nutr 50:37-81

20. Le Masurier GC (2004) Health-related physical fitness and physical activity trends among American youth. International Journal of Physical Education. Vol. XLI, Issue 2, 2 second Quarter 2004. Hofmann, Schorndorf
21. Bös K (2001) Bündnis "gesunde Kinder" - Unsere Kinder brauchen mehr Bewegung, Spiel und Sport, http://www.rz.uni-karlsruhe.de/ sportwiss/ Personal/Boes/GesundeKinder.pdf

22. Richter M, Settertobulte W (2003) Gesundheitsund Freizeitverhalten von Jugendlichen. In: Hurrelmann K, Klocke A, Melzer W, Ravens-Sieberer U (Hrsg) Jugendgesundheitssurvey internationale Vergleichsstudie im Auftrag der Weltgesundheits organisation WHO. Juventa, Weinheim, S 99-158

23. Bös K, Brochmann C, Eschette H et al. (2006) Gesundheit, motorische Leistungsfähigkeit und körperlich-sportliche Aktivität von Kindern und Jugendlichen in Luxemburg - Eine Untersuchung für die Altersgruppen 9, 14 und 18 Jahre. Abschlussbericht zum Forschungsprojekt . Luxembourg: SCRIPT

24. NASPE (2004) Physical activity for children: a statement of guidelines for children ages 5-12, 2nd edn. NASPE Publications, Reston, VA

25. President's Council on Physical Fitness \& Sports (2004) The President's challenge physical activity and fitness awards program. Bloomington, In: President's Council on Physical Fitness and Sports, S 9

26. Australian Government Department of Health and Ageing (2004) http://www. health.gov.au vom 16.6 .2006

27. WHO (2005) Global Strategy on diet, nutrition and physical activity, 2004; Why move for Health. Eigenverlag, Genf

28. Andersen LB, Harro M, Sardinha LB et al. (2006) Physical activity and clustered cardiovascular risk in children: a cross-sectional study (The European Youth Heart Study). Lancet 368:299-304

29. Biddle S, Nigg CR (2000) Theories of exercise behaviour. Internat J Sport Psychol 31:290-304

30. Hylton K, Bramham P, Jackson D, Nesti M (2001) Sports development: policy, process and practice. Routledge, London

31. Sallis JF, Owen N (1999) Physical activity \& behavioral medicine. Sage, Thousands Oaks

32. Dishman RK, Washburn RA, Heath GW (2004) Physical activity epidemiology. Human Kinetics, Champaign

33. Sygusch R (2005). Jugendsport - Jugendgesundheit. Ein Forschungsüberblick. Bundesgesundheitsbl Gesundheitsforsch Gesundheitsschutz 48:863-872

34. Fuchs R (1990) Sportliche Aktivität bei Jugendlichen. Entwicklungsverlauf und sozial-kognitive Determinanten. bps, Köln

35. Kurz D, Sonneck P (1996) Die Vereinsmitglieder Formen und Bedingungen der Bindung an den Sportverein. In: Kurz D, Sack HG, Brinkhoff KP (Hrsg) Kindheit, Jugend und Sport in NordrheinWestfalen. Der Sportverein und seine Leistungen. Materialien zum Sport in Nordrhein-Westfalen. Ministerium für Stadtentwicklung, Kultur und Sport des Landes Nordrhein-Westfalen, Düsseldorf, $S$ 75-159

36. Bös K (2001) Handbuch motorischer Tests. Hogrefe, Göttingen

37. Starker A, Lampert T, Worth A et al. (2007) Motorische Leistungsfähigkeit. Ergebnisse des Kinderund Jugendgesundheitssurveys (KiGGS). Bundesgesundheitsbl Gesundheitsforsch Gesundheitsschutz 50:775-783

38. Bös K, Worth A, Opper E et al. (2007) Motorik-Modul: Motorische Leistungsfähigkeit und körperlichsportliche Aktivität. Forschungsendbericht (in Vorbereitung) 
39. Lampert T, Mensink GBM, Romahn N, Woll A (2007). Körperlich-sportliche Aktivität von Kindern und Jugendlichen in Deutschland. Ergebnisse des Kinder- und Jugendgesundheitssurveys (KiGGS). Bundesgesundheitsbl Gesundheitsforsch Gesundheitsschutz 50:634-642

40. Oberger J, Romahn N, Opper E et al. (2006) Untersuchung zur motorischen Leistungsfähigkeit und körperlich-sportlichen Aktivität im Rahmen des Kinder- und Jugendgesundheitssurveys des Robert Koch-Institutes. In: Wydra G, Winchenbach $\mathrm{H}$, Schwarz M, Pfeifer K (Hrsg) Assessmentverfahren in Gesundheitssport und Bewegungstherapie. Czwalina, Hamburg, S 44-55

41. Helfrich C, Karger C (2006) Validierung eines Fragebogens zur Erfassung der körperlich-sportlichen Aktivität mittels SenseWear Armband. Unveröffentlichte Masterarbeit, Institut für Sport und Sportwissenschaft, Universität Karlsruhe (TH)

42. Lange M, Kamtsiuris P, Lange C et al. (2007) Messung soziodemographischer Merkmale im Kinderund Jugendgesundheitssurvey (KiGGS) und ihre Bedeutung am Beispiel der Einschätzung des allgemeinen Gesundheitszustands. Bundesgesundheitsbl Gesundheitsforsch Gesundheitsschutz 50:578-589

43. Schenk L, Ellert U, Neuhauser H (2007) Kinder und Jugendliche mit Migrationshintergrund in Deutschland. Methodische Aspekte im Kinderund Jugendgesundheitssurvey (KiGGS). Bundesgesundheitsbl Gesundheitsforsch Gesundheitsschutz 50:590-599

\section{Investieren, Forschen, Handeln: 125 Jahre nach der Entdeckung des Tuberkulose-Erregers durch Robert Koch}

Die Tuberkulose ist mit jährlich fast zwei Millionen Todesfällen weltweit nach wie vor eine der größten Gesundheitsgefahren. „Die vorrangige Herausforderung in der Bekämpfung dieser ansteckenden Krankheit liegt derzeit in Osteuropa" sagte Ulla Schmidt, Bundesministerin für Gesundheit, bei der Eröffnung eines internationalen Symposiums am 22. März 2007 anlässlich des Welttuberkulosetags am 24. März. „Vor allem die Entwicklung der Medikamentenresistenzen ist Besorgnis erregend", unterstreicht die Ministerin. Um die Tuberkulosekontrolle in Europa auf der politischen Ebene zu stärken, wird das Bundesministerium für Gesundheit gemeinsam mit dem WHO-Regionalbüro Europa am 22.10.2007 in Berlin ein Ministerforum mit Experten aus Gesundheit, Entwicklungshilfe und Forschung veranstalten. Das BMG unterstützt die Modernisierung des Gesundheitswesens insbesondere in Osteuropa seit Jahren.

In Deutschland hat das Robert KochInstitut nach dem auf dem Symposium vorgestellten „Tuberkulose-Bericht für 2005“ 6.045 Tuberkulose-Erkrankungen registriert. „Bei der Schwere der Erkrankung und einer Therapiedauer von mindestens sechs Monaten ist das keine kleine Zahl" betont Reinhard Kurth, Präsident des Robert Koch-Instituts. "Darin zeigt sich auch die hohe Bedeutung des Öffentlichen Gesundheitsdienstes." Insgesamt 188 Patienten starben 2005 an den Folgen der Infektionskrankheit.

Am meisten gefährdet sind die sozial Schwächeren. Schon Robert Koch unterstrich die große Bedeutung der notwendigen Verbesserung der sozialen Strukturen und der staatlich zu fördernden Tuberkulosekontrolle. Bei Erkrankungen mit resistenten Bakterienstämmen, die unempfindlich gegen die verfügbaren Medikamente sind, ist die Behandlung sehr viel langwieriger und teurer. In Deutschland ist der Anteil von multiresistenten Erregern (gleichzeitige Resistenz gegenüber Isoniazid und Rifampicin) mit 2,7 \% gegenüber den Vorjahren weiter gestiegen (2004: 2,5\%, 2003: 2,1 \%; 2002: 2,1\%). In Osteuropa und Zentralasien liegen die Raten bei circa $15 \%$ der Neu- erkrankungen, dreifach höher als in jeder anderen Region weltweit.

Daher unterstützt das von Sabine RüschGerdes geleitete Nationale Referenzzentrum für Mykobakterien in Borstel den Aufbau von Labornetzwerken in besonders betroffenen Staaten und hilft bei der Weiterbildung osteuropäischer Ärzte. Vor Ort in den betroffenen Länder engagieren sich auch die Deutsche Lepra- und Tuberkulosehilfe (DAHW), das Deutsche Rote Kreuz (DRK) und das im vergangenen Jahr gegründete KochMetschnikow-Forum (KMF), eine deutschrussische Initiative des Petersburger Dialoges, die Kooperationsprojekte auf dem Gebiet der Bekämpfung der Infektionskrankheiten koordiniert und gestaltet. „Die TuberkuloseBekämpfung muss auf allen Ebenen gestärkt werden und darf angesichts der - unverzichtbaren - Bemühungen um die Eindämmung von HIV nicht vergessen werden", fordern DAHW, DRK und KMF.

Quelle: RKI, Redaktion:Springer-Verlag 